\title{
Simulating Electron Transport and Synchrotron Emission in Radio Galaxies: Shock Acceleration and Synchrotron Aging in Axis-symmetric Flows ${ }^{4}$
}

\author{
T. W. Jones ${ }^{1}$, Dongsu Ryu ${ }^{2}$ and Andrew Engel ${ }^{1,3}$
}

\begin{abstract}
We introduce a simple and economical but effective method for including relativistic electron transport in multi-dimensional simulations of radio galaxies. The method is designed to follow explicitly diffusive acceleration at shocks, and, in smooth flows, second-order Fermi acceleration, plus adiabatic and synchrotron losses for electrons in the energy range responsible for radio emission in these objects. We are able to follow both the spatial and energy (momentum) distributions of the electrons, so that direct synchrotron emission properties can be modeled in time dependent simulated flows of this type for the first time. That feature is essential if simulations are to bridge successfully the fundamental physical gap between flow dynamics and observed emissions.
\end{abstract}

As an initial step towards that goal, we present results from some axis-symmetric MHD simulations of Mach 20 light jet flows. These explicitly explore the effects of shock acceleration, as well as adiabatic expansion and synchrotron aging in smooth flows. The simulations demonstrate the importance of the fact that even for steady inflows jet terminal shocks are not simple, steady plane structures. Most importantly this should play a very major role in determining the properties of synchrotron emission within the terminal hot spot and in the lobes generated by the jet back flow. In fact, the outflows are inherently complex, because of the basic "driven" character of a jet flow. Consequently, the nonthermal electron population emerging from the jet may encounter a wide range of shock types and strengths, as well as magnetic field environments.

We may expect to find a complex range in synchrotron spectral and brightness patterns associated with terminal hot spots and lobes. These include the possibility of steep spectral gradients (of either sign) within hot spots, the potential in lobes for islands of flat spectrum electrons within steeper spectral regions (or the reverse) and spectral gradients coming from the dynamical history of a given flow element rather

\footnotetext{
${ }^{1}$ Department of Astronomy, University of Minnesota, Minneapolis, MN 55455: twj@msi.umn.edu

${ }^{2}$ Department of Astronomy \& Space Science, Chungnam National University, Daejeon 305-764, Korea: ryu@canopus.chungnam.ac.kr

${ }^{3}$ Department of Physics, University of Arizona Tucson, AZ 85721: aaengel@physics.arizona.edu

${ }^{4}$ Accepted for publication in the Astrophysical Journal
} 
than from synchrotron aging of the embedded electrons. Finally, synchrotron "aging" in the lobes tends to proceed more slowly than one would estimate from regions of high emissivity. That is a consequence of the fact that those regions are ordinarily places where the magnetic fields are the strongest, so that the instantaneous rates of energy loss are atypical of the full history of the electron population. This feature supports earlier suggestions that nonuniform field structures may help to explain why dynamical ages of FRII sources often seem to be greater than the apparent age of the electrons radiating in the lobes, as measured in terms of spectral steepening, or absence thereof.

Subject headings: particle acceleration - galaxies: jets - magnetohydrodynamics: MHD - radio continuum: galaxies 


\section{Introduction}

The standard paradigm for radio galaxies (RGs) is based on high speed plasma jets, formed in active galaxy nuclei (AGN), but penetrating far into circumgalactic environments and creating giant lobes of luminous material as a consequence of interactions with the intergalactic medium (e.g., Bridle 1992). The defining radio emission represents synchrotron radiation from relativistic electrons and magnetic fields. Those two crucial constituents are thought to be transported from the AGN, enhanced or generated by the jet-IGM encounter or both. Modern radio interferometry has provided richly detailed intensity, spectral and polarimetric images of RGs (e.g., Leahy et al. 1997), while rapidly advancing computational tools have allowed increasingly sophisticated multi-dimensional magnetohydrodynamical (MHD) simulations of the plasma flows (e.g., Clarke 1996). This paper presents the first such simulations that explicitly include time dependent transport of the relativistic electrons that produce the observed radio emission in such objects. Coleman \& Bicknell 1988 carried out an early related calculation by mapping an analytic electron distribution onto a simulated bow-shock flow pattern, while Jones \& Kang 1993 followed the time dependent behavior of a simplified electron distribution during the evolution of a shocked gas cloud. Clarke et al. 1989 and Matthews \& Scheuer 1990 computed early models of synchrotron emission from RGs based on simulated fluid dynamical variables and intelligent guesses at the possible relativistic electron properties. But, no published simulations have followed the full electron distribution through a time-dependent evolution in a simulated RG-like flow. That important feature is a very difficult technical challenge, because the length and time scales needed to model effectively the microphysics of electron transport are typically many orders of magnitude smaller than those convenient to include in full scale hydrodynamical or magnetohydrodynamical simulations of RGs.

To avoid these difficulties we introduce here a simple, economical method of relativistic electron transport that directly utilizes those mismatched scales and is suitable for time-dependent RG simulations. The method is designed to include the effects of diffusive shock acceleration and second-order Fermi acceleration, as well as adiabatic compression or expansion and synchrotron radiative cooling of the electrons in smooth flows. We present results of its initial application. There are many dynamical effects that are likely to influence the eventual synchrotron brightness distribution and spectrum to be expected in realistic models of RGs. Before it makes sense to attempt anything that could be termed a "real" model of a RG, it is crucial that the individual dynamical influences be understood. The present paper, therefore, is intended to begin addressing these individual factors. The role of shock acceleration of RG electrons is centrally important (e.g., Heavens \& Meisenheimer 1987; Blandford \& Eichler 1987; Takarada 1989; Krülls 1992) While there are many studies of the physics of particle acceleration at plane or spherical shocks (e.g., Kang \& Jones 1991), there are few previous simulations designed to explore shock

acceleration in complex flows (but see e.g., Jones, Kang \& Tregillis 1994) and, as mentioned, none for flows similar to those expected in RGs. Therefore, our first priority is to explore how shock acceleration may be characterized and recognized in such environments, or the degree to which 
such flows may tend to confuse simple interpretations of this process.

Given these objectives and the fact that 2D, axis-symmetric flows are much easier to understand than 3D flows and still provide a first cut at the properties of RG flows, we limit this initial study to that problem. We have begun an extension of this work into fully 3D simulations and will present those results elsewhere. Since synchrotron cooling (commonly called "aging") is also likely to be a significant effect and will compete with and mask the influence of shock acceleration, we also include here an example of a flow that includes both of these effects together. We defer to later papers inclusion of second-order Fermi acceleration, not because it is necessarily unimportant, but because it is significantly more difficult to establish a unique, physical characterization of the momentum diffusion controlling it. Thus, at this stage, it would add significant complexity and uncertainty to an already complex set of questions.

The plan of the paper is this. In $\S 2$ we outline our methods, including the new electron transport scheme. Section 3 introduces the parameters of the jet flows we have modeled, while $\S 4$ discusses our results. A brief summary of key findings is given in $\S 5$. An appendix contains a detailed discussion of practical requirements for numerical treatment of electron transport in RGs and justification for the scheme we introduce here.

\section{Methods}

\subsection{Dynamics}

We evolve the equations of ideal nonrelativistic magnetohydrodynamics (MHD) in cylindrical coordinates $(r, \phi, z)$, with $\phi$ an ignorable coordinate. All three components of velocity and magnetic fields are included, so the model is $2 \frac{1}{2}$-D. The code is an MHD extension of Harten's (Harten 1983) conservative, second-order finite difference "Total Variation Diminishing" scheme, as detailed in Ryu \& Jones 1995; Ryu, Jones \& Frank 1995; Ryu, Yun \& Choe 1995 and Ryu et al. 1998. The code preserves $\nabla \cdot B=0$ at each time step using an approach similar to the Constrained Transport (CT) scheme (Evans \& Hawley 1988) as described in detail by Ryu et al. 1998a. We use a passive "mass fraction" or "color" tracer, $C_{j}$, to distinguish material entering the grid through the jet orifice $\left(C_{j}=1\right)$, or from ambient plasma $\left(C_{j}=0\right)$.

\subsection{Electron Transport}

Our electron transport scheme is an adaptation of the standard diffusion-convection equation for charged particles (e.g., Skilling 1975);

$$
\frac{\partial f}{\partial t}=\frac{1}{3} p \frac{\partial f}{\partial p}(\nabla \cdot \vec{u})-\vec{u} \cdot \nabla f+\nabla \cdot(\kappa \nabla f)+\frac{1}{p^{2}} \frac{\partial}{\partial p}\left(p^{2} D \frac{\partial f}{\partial p}\right)+Q
$$


where $f(\vec{x}, p, t)$ is the isotropic part of the nonthermal electron distribution, $\kappa$ is the spatial diffusion coefficient, $D$ is the momentum diffusion coefficient, $Q$ is a source term that represents the net effects of "injection" and radiative losses at a given momentum, $p$, while the thermal plasma velocity is $\vec{u}$. This equation is valid for "fast" (superthermal) particles when scattering is strong enough to keep the particle distribution almost isotropic and is appropriate on lengths large compared to the particle scattering length itself.

The first term on the right accounts for adiabatic compression or rarefaction in the background flow. Eq. 2-1] is not valid within shocks, but when integrated across a velocity discontinuity, the first three terms account for "first order Fermi acceleration" at shocks, also known as "diffusive shock acceleration". The fourth term allows for second-order Fermi acceleration resulting from particle interaction with Alfvénic turbulence (e.g., Skilling 1975; Blandford \& Eichler 1987).

As mentioned in the introduction and explained in detail in the Appendix, there is a severe mismatch between RG dynamical scales and diffusive transport scales that apply to eq. [2-1] for electron momenta relevant to radio synchrotron emission. That mismatch makes it impractical with conventional computational methods to solve eq. [2-1] as it stands to study electron transport in full RG flows. That is, the electron diffusive lengths and times are vastly smaller than those appropriate to the gasdynamics. The key physical fact is that the characteristic energy of electrons radiating synchrotron emission can be stated roughly as $E \sim 3 \nu_{9}^{\frac{1}{2}} / B_{10}^{\frac{1}{2}} \mathrm{GeV}$, where $\nu_{9}$ is the observed frequency in $\mathrm{GHz}$, and $B_{10}$ is the magnetic field compared to $10 \mu \mathrm{G}$ or $\mathrm{nT}$. Thus, the electrons of interest mostly have energies $\lesssim 10 \mathrm{GeV}\left(p \lesssim 10^{4} \mathrm{mc}\right)$. The gyroradius of such particles is $r_{g} \sim 3 \times 10^{11} E_{G e V} / B_{10} \mathrm{~cm}$. This leads to characteristic diffusion lengths and shock acceleration times in RGs $\lesssim \mathrm{AU}$ and $\lesssim \mathrm{yr}$, respectively compared to flow scales typically measured in kpc and kyr or larger. The most serious consequence of this comes from the requirement that numerical shocks must appear thinner than a particle diffusion length for accurate solutions of eq. [2-1] (see the Appendix).

However, as also explained in the Appendix, that same mismatch can be exploited to develop a simplified equation of electron transport, provided $f(p)$ is sufficiently broad that it can be represented as a piecewise power law over finite momentum bins. This last feature is very natural, in fact, in light of the same small time and length scales for electrons, since very rapid diffusive shock acceleration guarantees that $\mathrm{GeV}$ electrons emerge from shocks with power law momentum distributions on time scales much less than time steps required by the MHD. For much higher electron energies these simplifying conditions break down, but the method we derive could be used to provide a "low energy injection spectrum" for those particles, as well.

For continuity with the remainder of our discussion we present here the simplified transport equation, but refer readers to the Appendix for derivation details and tests of its validity. We divide the momentum range of interest into a modest number, $\mathrm{N}$, of logarithmically spaced bins bounded by $p_{0}, \ldots, p_{N}$. Defining $y_{i}=\ln p_{i} / p_{0}$, bin widths can be expressed as, $\Delta y_{i}=y_{i+1}-y_{i}=\ln p_{i+1} / p_{i}$. We can integrate equation [2-1] within each momentum bin to define $n_{i}=4 \pi \int_{p_{i}}^{p_{i+1}} p^{3} f d \ln p$ as the 
number of electrons in the bin. For convenience we normalize $n_{i}$ by the total plasma mass density, to form $b_{i}=n_{i} / \rho$, then assume a piecewise power law $f(p)=f_{i}\left(p / p_{i}\right)^{-q_{i}}$ within $p_{i} \leq p \leq p_{i+1}$, so that $n_{i}$ is given in terms of $f_{i}, q_{i}$ and $\Delta y_{i}$ by eq. [A2]. For these relatively low energies diffusive transport at shock discontinuities is properly handled by defining the form of the electron distribution just down stream of a shock to be the steady state power law appropriate to the jump conditions for that shock. That fixes the ratio $b_{i+1} / b_{i}$ at shocks. Electrons are injected from the thermal plasma at shocks using a common injection model, so that a fixed fraction, $\epsilon$, of the total electron flux through a shock is injected and accelerated to the appropriate power law momentum distribution. Away from shocks, in smooth flows, but where the diffusion lengths of the electrons are much smaller than dynamical lengths, spatial diffusion is negligible. We can easily include the effects of synchrotron "aging". When all of these features are included eq. 2-1] becomes (see also eq. A2])

$$
\frac{d b_{i}}{d t}=\left.4 \pi\left(\frac{1}{3} \nabla u-\frac{q D}{p^{2}}+\frac{1}{\tau_{s o}} \frac{p}{\hat{p}}\right) \frac{p^{3} f}{\rho}\right|_{p_{i}} ^{p_{i+1}}
$$

where $\tau_{s o}=\frac{3}{4} \frac{(m c)^{2}}{\sigma_{T} U_{B}} \frac{1}{\hat{p}}$ defines the synchrotron cooling time at momentum $\hat{p}$, which we take arbitrarily to be $\hat{p}=10^{4}$ mc. In this expression, $\sigma_{T}$ is the Thomson cross section, and $U_{B}=B^{2} /(8 \pi)$. Note that the right hand side of eq. 22-2 is the difference between fluxes at the two momentum boundaries of the bin $i$. Thus, we have a conservative, finite volume scheme that depends on a simple model of sub-grid structure (in momentum space) for its accuracy.

Although straightforward to include, we now drop the second order Fermi term, " $D$ " from these initial simulations for the following reasons. First, in the vicinity of shocks, this acceleration process is likely to be much less efficient than first-order Fermi acceleration. That is simply because the second-order process comes from nearly balanced energy fluxes of waves propagating parallel and antiparallel to the magnetic field that resonantly scatter with the electrons; i.e., on "isotropic" Alfvénic turbulence. First-order Fermi acceleration, on the other hand, depends only on waves propagating in the streaming direction that will be generated by the streaming particles themselves. In either situation resonant waves have wavelengths comparable to the particle gyroradius. Downstream of the shocks where particle streaming may be less important, it is significant for such low particle energies that the resonant wavelengths are probably within a couple orders of magnitude of thermal ion gyroradii (taking $u_{s} \sim 10^{8} \mathrm{~cm} \mathrm{~s}^{-1}$ ). Such waves may be fairly strongly dissipated, for example, by nonlinear Landau damping (e.g., Lee \& Völk 1973). Thus the level of relevant isotropic Alfvénic turbulence will likely depend on a local cascade of hydrodynamical turbulence (e.g., Grappin et al. 1982). While we will certainly want to understand what role post-shock, second-order acceleration might play (e.g., Borovsky \& Eilek 1986; Krülls 1992), only ad hoc models could be applied at present. Since this set of computations represents the first effort to treat nonthermal electron transport inside time dependent models of RGs, and since there are many possible competing influences to be understood before "realistic" models are sensible, the best initial strategy is to restrict ourselves to the most straightforward physics that is likely to be important. First-order acceleration is efficient and almost certain to happen at shocks, so its role must be understood from the beginning. 


\subsection{Computation of Synchrotron Emissivity}

From the spatial distribution of $b_{i}$ and $q_{i}$ along with the MHD variables, $\rho$ and $B$, it is straightforward to compute the synchrotron spectral emissivity, $j_{\nu}$. Ignoring corrections due to slow curvature in the electron spectrum, $j_{\nu}$, can be expressed as (cf. Jones, O'Dell \& Stein 1974)

$$
j_{\nu}=j_{\alpha o} \frac{4 \pi e^{2}}{c} f(p) p^{q}\left(\frac{\nu_{B_{\perp}}}{\nu}\right)^{\alpha} \nu_{B_{\perp}}
$$

with $\alpha=\frac{q-3}{2}, j_{\alpha o} \sim 1$ (cf. Jones, O'Dell \& Stein 1974) and $\nu_{B_{\perp}}=\nu_{B} \cos \theta$ the electron cyclotron frequency in terms of the magnetic field projected over the angle $\theta$ onto the plane of the sky. $f(p)$ along with $q$ are found from mapping $b_{i}$ and $q_{i}$ onto the critical synchrotron frequency as

$p=m c \sqrt{\frac{2 \nu}{3 \nu_{B}}}$. We have interpolated $q$ between momentum bin centers to give $\alpha$ a continuous form. Eq. [2-3] would break down for strongly curved spectra, but by that point in calculations of the type used here the local index will be too steep for the emission to be significant. In a calculation designed to predict the surface brightness distribution of a RG model one should include explicitly the effects of variations in $\cos \theta$. That is not our intention here, and such a calculation would need to evaluate carefully the effects of "sub-grid" fluctuations in the field directions and of the orientation of the jet with respect to the line of sight. Our aim here is to understand how electron acceleration and transport are likely to be reflected in the overall emission properties of a region within a RG, independent of the location of the observer. For those purposes it is better to ignore the factor $\cos \theta$, so will in this paper simply take it to be unity. Again, we are not in this paper computing surface brightness distributions from these simulations, which would involve line-of-sight integrations of $j_{\nu}$. That would be inappropriate, given the axis-symmetric nature of the simulations, and would further exaggerate the dynamical constraints imposed by this symmetry.

\section{The Simulated Jet Properties}

Our initial simulated MHD jets are all dynamically identical. They have a simple, "top hat" velocity profile, are Mach 20 with respect to the uniform ambient medium $\left(M_{j}=u_{j} / c_{a}=20\right)$, in thermal pressure balance with it, and have a density contrast $\eta=\rho_{j} / \rho_{a}=0.1$. The jet enters at $z=0$, with an initial radius of 36 zones, while the entire uniform grid is $384 \times 1536$ zones $\left(10 \frac{2}{3} r_{j} \times 42 \frac{2}{3} r_{j}\right)$. Defining length and time in units of initial jet radius $\left(r_{j}=1\right)$ and ambient sound speed $\left(c_{a}=\sqrt{\gamma P_{a} / \rho_{a}}=1\right.$, with $\left.\gamma=\frac{5}{3}\right)$, the simulations are followed for about 11.7 time units, when the bow shock of the jet reaches the right $z$ boundary. Reflecting boundaries are used along the jet axis, while continuous boundaries are used elsewhere. There is a background poloidal magnetic field ( $\left.B_{\text {background }}\right)\left(B_{\phi}=B_{r}=0 ; B_{z}=B_{z o}\right.$ ), with a magnetic pressure $1 \%$ the gas pressure; i.e., $P_{b}=\frac{1}{\beta} P$, with $\beta=10^{2}$. The in-flowing jet carries an additional toroidal magnetic field component appropriate to a uniform axial jet current with a return current on the surface of the jet; i.e., $B_{\phi}=2 \times B_{z o}\left(r / r_{j}\right)$ for $r \leq r_{j}$. At $r=r_{j}, \beta=20$. The in-flowing jets are slightly 
over-pressured at the outside.

These simulations are truly MHD rather than HD, despite the apparent weakness of the initial magnetic field. Even at the initial strengths modeled here the magnetic fields play a role in the evolution, especially in the cocoon. There are numerous locations where the plasma $\beta \sim 10$, so that its pressure is not entirely ignorable. There is, however, a more important and much less recognized role for the field in flows initialized with even $\beta>>1$. In 2-D and 3-D MHD simulations of the Kelvin-Helmholtz instability, for example, we have demonstrated crucial dynamical contributions from magnetic fields in flows with initial $\beta>10^{3}$ (e.g., Jones et al. 1997; Ryu et al. 1998b; Jones, Ryu \& Frank 1998). The point is that through field "stretching", magnetic tension can be increased in complex flows to the point where it is comparable to or exceeds the Reynolds stresses of the gasdynamical flow ( coming from spatial variations in $\rho u_{i} u_{j}$ ), even though the magnetic pressure may be smaller than the gas pressure. The relative importance of Maxwell to Reynolds stresses can be roughly represented by the Alfvén Mach number in a flow, $M_{A}=u / v_{A}$. When the local $M_{A}$ drops to small values in a complex flow, that can lead through reconnection to so-called "dynamical alignment" and flow "self-organization". There are also many locations within our model flows where $M_{A} \lesssim 1$. In short, the flows are made smoother by the presence of the field, even though in the original configuration the field nominally appeared too weak to influence the dynamics. A comment may be in order on the meaning of magnetic reconnection obtained from an "ideal MHD" code. While reconnection is most fundamentally a topological transition (e.g., , Axford 1984), it cannot take place without resistive, dissipative effects on small scales. In an ideal MHD code these dissipative effects are numerical in origin, so we cannot model the microphysics of driven reconnection (which is still not understood at this time, in any case). However, there is evidence that the phenomenology of reconnection is often correctly captured. For example, Miniati et al. (1998), using the Cartesian version of the code we employ here, demonstrate clear development in 2-D supersonic MHD cloud simulations of the so-called "resistive tearing-mode" instability that is physically associated with driven reconnection.

We present here three examples of the electron transport within the dynamics of the above jet flows. Their properties are listed in Table 1. Electrons are modeled in the momentum range $p_{0}=m_{e} c$ and $p_{N} \approx 1.63 \times 10^{5} m_{e} c$. All three include the effects of diffusive shock acceleration. Models 1 and $\mathbf{2}$ are alike in that the electrons have negligible influence from synchrotron aging , while Models $\mathbf{1}$ and $\mathbf{3}$ are alike in that the electron populations originate entirely with the in-flowing jet. Model 2 differs in that the electron population is mostly injected locally from thermal plasma at shocks within the modeled flow. Model $\mathbf{3}$ differs, then, in that electrons are influenced very significantly by synchrotron aging.

In the "adiabatic" Models $\mathbf{1}$ and 2, there is very little curvature to the electron spectra, so in those models we used four momentum bins (i.e., $N=4$ ) giving $\ln \frac{p_{i+1}}{p_{i}}=3$. (See the Appendix for justification of these numbers.) In Model 2 we injected electrons at $p_{0}$ using the simple model described in $\S$ A.3. Electrons should be injected at slightly suprathermal postshock energies, so the value $p_{0}=m_{e} c$ was selected as appropriate for jets with speed $u_{j} \sim 0.1 c$, assuming that shocked 
Table 1. Summary of Simulations

\begin{tabular}{ccccccc}
\hline \hline & & \multicolumn{3}{c}{} & & \\
\cline { 3 - 4 } Model $^{\mathrm{a}}$ & $\mathrm{N}^{\mathrm{b}}$ & $\begin{array}{c}\text { In-flowing Electrons }^{\mathrm{c}} \\
\left(b_{1}\right)\end{array}$ & $\begin{array}{c}\text { Jet Spectrum }^{\mathrm{d}} \\
q_{\text {jet }}\left(\alpha_{\text {jet }}\right)\end{array}$ & $\begin{array}{c}\text { Shock Injection }^{\mathrm{e}} \\
(\epsilon)\end{array}$ & $\begin{array}{c}\text { Cooling Time }^{\mathrm{f}} \\
\left(\tau_{\text {so }}\right)\end{array}$ & $\begin{array}{c}B_{\text {background }^{\mathrm{g}}} \\
\mu \text { Gauss }\end{array}$ \\
\hline 1 & 4 & $10^{-4}$ & $4.4(0.7)$ & 0.0 & 40 & 10 \\
2 & 4 & $10^{-6}$ & $4.4(0.7)$ & $10^{-4}$ & 40 & 10 \\
3 & 8 & $10^{-4}$ & $4.4(0.7)$ & 0.0 & 4 & 30 \\
\hline
\end{tabular}

${ }^{a}$ All models used Mach 20 jets $\left(M_{j}=u_{j} / c_{a}=20\right)$, with a density contrast, $\eta=\rho_{j} / \rho_{a}=0.1$. Units come from a jet radius, $r_{j}=1$, an ambient density, $\rho_{a}=1$, and a background sound speed, $c_{a}=\sqrt{\gamma P_{a} / \rho_{a}}=1(\gamma=5 / 3)$. The cylindrical computational box was $10.67 r_{j}$ units high and $42.67 r_{j}$ long. End time for each simulation was $t=10.67$ units. There was a uniform, poloidal jet magnetic field, $B_{z 0}$ that also extended to the background, so $B_{z 0}=B_{\text {background }}$ with $\beta=P / P_{b}=100$, while the incoming jet also contained a toroidal field, $B_{\phi}=2 \times B_{z 0}\left(r / r_{j}\right)$.

${ }^{\mathrm{b}}$ Number of electron logarithmic momentum bins spanning the range $p_{0}=1 \mathrm{mc}$ to $p_{N}=1.63 \times 10^{5} \mathrm{mc}$. $\left(\ln p_{N} / p_{0}=12\right)$

${ }^{\mathrm{c}}$ Ratio of nonthermal to thermal electrons in the incident jet flow.

${ }^{\mathrm{d}} q_{j e t}$ is the momentum distribution power law index of the in-flowing electron population; $\alpha_{j e t}$ is the corresponding synchrotron index.

${ }^{\mathrm{e}}$ Assumed fraction of the thermal electron population injected to the nonthermal population as it passes through a shock.

${ }^{\mathrm{f}}$ Time for electrons to cool below momentum, $\hat{p}=10^{4} \mathrm{mc}$ in the ambient, or background, magnetic field, measured in time units $r_{j} / c_{a}$.

${ }^{\mathrm{g}}$ Field that would produce the listed cooling time, assuming illustrative values for the simulated jet: $u_{j}=0.1 c$, $r_{j}=1 \mathrm{kpc}$. This would also map the displayed synchrotron emissivity data onto $1.4 \mathrm{GHz}$. Applying the additional constraints on the jet listed above, the implied jet density and kinetic luminosity are $\rho_{j} \approx 3 \times 10^{-29} B_{\text {background }}^{2} \mathrm{~g}$ $\mathrm{cm}^{-3}$ and $K_{\text {kinetic }}=\frac{\pi}{2} \rho_{j} u_{j}^{3} r_{j}^{2} \approx 1.2 \times 10^{43} B_{\text {background }}^{2}$ erg sec ${ }^{-1}$, with $B_{\text {background }}$ in $\mu$ Gauss. 
electrons are thermalized to a temperature comparable to but smaller than the ions. Since the nonthermal, cosmic-ray electrons are passive, all results could be scaled for different choices of $p_{0}$.

To include synchrotron aging it is necessary to relate the characteristic cooling time $\tau_{s o}$ defined in association with eq. A3 to the computational time units, here given by $r_{j} / c_{a}$. The cooling time depends as well on the magnetic field strength and the momentum $p$ as

$$
\tau_{s}=2.5 \times 10^{1} \frac{1}{p_{4}} \frac{u_{j 8}}{M_{j}} \frac{1}{r_{j k}} \frac{1}{B_{10}^{2}},
$$

where $\tau_{s}=\tau_{s o} \frac{\hat{p}}{p}, p_{4}$ is the electron momentum in units $10^{4} \mathrm{mc}, u_{j 8}$ is the jet speed expressed in units of $10^{8} \mathrm{~cm} \mathrm{~s}^{-1}, M_{j}$ is the jet Mach number (here set to $M_{j}=20$ ), $r_{j k}$ is the incoming jet radius in $\mathrm{kpc}$, and $B_{10}$ is the strength of the magnetic field in units of $10 \mu \mathrm{G}$, or equivalently in units nT. By comparison, the end points of our simulations are $t=10 \frac{2}{3}$.

For Models 1 and 2 we have set $\tau_{s o}=40$, the objective being to produce negligible aging for electrons of interest during the simulation. To project emission from electrons with momenta $p \lesssim 10^{4} \mathrm{mc}$ into the radio band, we should be considering magnetic fields $\gtrsim 10 \mu \mathrm{G}$. Assuming, for example, that $B_{z 0}=10 \mu \mathrm{G}$, and $u_{j 8}=30$, then $r_{j k} \approx 1.1$, so that the length of our computational box would be about $45 \mathrm{kpc}$, and the time unit, $r_{j} / c_{a} \approx 7 \times 10^{5}$ years.

By contrast, for Model 3 we set $\tau_{s o}=4.0$, so that synchrotron aging is now very significant for electrons in the energy range of interest over the time scale of the simulation. Keeping the same physical jet speed and radius, as well as the time unit, this case would correspond to an axial magnetic field $B_{z 0} \approx 30 \mu \mathrm{G}$. Other associated dependent parameters are outlined in Table 1. These combinations are illustrative only and not meant to be particularly representative of any real RGs. Other combinations would work as well for our present purposes, which are aimed at the physics of the electron transport. For the simulation of Model 3, we used eight momentum bins $(N=8)$ to cover the same range as the other models, as justified in $\S \mathrm{A} .4$.

\section{Discussion}

\subsection{Flow Dynamics}

We first outline some key dynamical properties of the simulations, remembering that the MHD properties of the different electron transport models are identical except for dimensional scaling factors. Fig. 1 shows at $t=10.67$ images of the plasma density, $\rho$, the magnetic field intensity, $B$, and gas compression, $\frac{d \ln \rho}{d t}=-\nabla \cdot \vec{u}$. The compression highlights shock structures. These images remind us that such flows are complex and unsteady. All of the structures are ephemeral or at least highly variable. Jets, as strongly driven flows are not reasonable to model as equilibrium structures (cf. Sato et al. 1996). As described by many before us (e.g., Norman, Smarr \& Winkler 1985; Lind et al. 1989), once equilibrium is broken, jet plasma alternately expands and then refocuses while interacting with its self-generated cocoon, creating oblique jet 
shocks as a result. Ð Those shocks are neither steady nor stationary, however. Most important to our discussion, the oblique jet shocks interact episodically with the terminal jet shock, causing it to vary in both strength and structure. The jet never re-establishes an equilibrium in its flow. The terminal shock also includes a "Mach stem", so that in 2-D at least, jet flow coming down the outside of the jet, near $r_{j}$, always exits through an oblique shock. At times there is little or no perpendicular terminal shock; then, most of the emerging jet flow is only weakly shocked. When a perpendicular portion to the terminal shock exists, it usually is strong, with a compression ratio $\sigma \approx 3.8(\rightarrow q \approx 4.08 ; \alpha \approx 0.54)$ in these simulations. The many other shocks and the oblique segments of the terminal shock are mostly weak (Fig. 1c), but they can exhibit density jumps, $\sigma \gtrsim 2-3\left(\rightarrow q \approx 6-\frac{9}{2}\right)$. Along the jet axis intersecting oblique shocks can be strong, but these intersections intercept a small fraction of the flow, so will not produce a large population of flat spectrum electrons.

Vorticity is shed from the outer edge of the terminal shock to form the turbulent jet cocoon. There are distinct episodes of strong "vortex shedding" coincident with disruption and reformation of the terminal shock. Once shed, the vortices interact with the Kelvin-Helmholtz unstable boundary layer of the jet, generating an even more complex back-flow and perturbing the jet flow, as well. All of these dynamical features are represented in the Fig. 1. snapshot. The large "rolls" visible in Fig. 1 are remnants of vortex rings that were shed earlier than the time displayed. The magnetic field in the back-flow is dominated within an axis-symmetric flow by $B_{\phi}$, both because this magnetic component is enhanced by stretching in the expanding flow emerging from the jet, and because magnetic reconnection of the $B_{r}, B_{z}$ field components annihilates most of that field from the back-flow in an axis-symmetric geometry. This flux annihilation inside vortical flows is well-known and sometimes termed "flux expulsion" in the MHD literature (Weiss 1966). In a 3-D flow both the vortex and magnetic field structures would be stretched, twisted and tangled; i.e., the back-flow would become turbulent and disordered on small scales (e.g., Norman 1996; Ryu et al. 1998b; Jones, Ryu \& Frank 1998) leading to vortex and magnetic flux tube complexes.

\section{2. $\quad$ Electron Transport \& Emissivity}

The electron transport we model takes place "on top of" this dynamics; i.e., , the electrons do not feed back dynamically on the flow. To check this for consistency we confirmed that for the numbers we assume, the electrons never represent more than about $0.1 \%$ of the kinetic energy density in the jet or the back flow. Remember that the dynamics behind the electron transport and synchrotron simulations discussed below is identical in each model. The electron distribution, and especially its momentum dependence, reflect the flow history of a given fluid element as appropriate for the particular transport assumptions, as well as the current fluid condition. Our

\footnotetext{
${ }^{5}$ In this paper "oblique" and "perpendicular" refer to the angle between the shock face and the velocity field, not the shock normal and the magnetic field, as is customary in the particle acceleration literature.
} 
objective in this paper is to begin to learn how to interpret the emergent patterns. Since the astronomical tool for this is synchrotron emission, we will explore the physics in that paradigm using local emissivities. We expect that this approach will make much more tractable the interpretation of full 3D simulations. For the convenience of the discussion we express emissivities as they would be at $1.4 \mathrm{GHz}$, based on the fiducial magnetic field values listed at the end of $\S 3$ and in Table 1. They can be easily rescaled by choosing other combinations of source parameters. Illustrative examples of the emissivity properties in these models are shown in Fig. 2 - 4. Fig. 5 explores the correlations between emissivity, spectral index and magnetic field strength using the same data displayed in the other figures. At the start we point out the obvious "limb brightening" of the emissivities seen in all of the models displayed. This feature is not characteristic of real radio lobe surface brightness in FRII sources, but is an artifact of the assumed axis-symmetry of the simulations; particularly the persistence of the large "rolls" in the back-flow and the decoupling that occurs in axis-symmetry between $B_{\phi}$ and $\left(B_{r}, B_{z}\right)$. In an equivalent 3-D simulation the bright structures should be better mixed into the turbulent interior regions of the back-flow. This is one reason we defer any display of model surface brightness until we have 3-D structures in the models. That does not detract from the present discussion, however, since this initial exploration is mostly aimed at identifying physical relationships in emission properties and flow properties, as well as recognizing emissivity characteristics that we may eventually be able to use as diagnostics of the history of the local electron population.

\subsection{1. "Adiabatic" Models 1 and $\mathbf{2}$}

Fig. 2a and $2 \mathrm{~b}$ provide grayscale images of $\log j_{\nu}$ at $1.4 \mathrm{GHz}$ for the "adiabatic" electron models $\mathbf{1}$ and $\mathbf{2}$ at $t=10.67$. For these two models emissivity values from eq. 22-3] are displayed when they are within a factor $3 \times 10^{3}$ of the peak emissivity. Smaller emissivities are blacked out. For comparison we also display with the same dynamic range in Fig. 2c a "pseudo emissivity", based on the MHD properties of the flow; i.e., $j_{c}=C_{j} P B^{\frac{3}{2}}$, modeled after the approach introduced in Clarke et al. 1989. We have added the jet color variable, $C_{j}$ to Clarke's original definition, because of differences in our assumed background magnetic field. Clarke's magnetic field vanished outside the jet flow and its cocoon, whereas we used a simpler model that continues the axial field into the background. Since $C_{j}=0$ in the background our definition allows $j_{c}>0$ only for regions containing jet plasma, so that they are comparable to Clarke's. On the other hand, for the Model 1, 2 and $\mathbf{3}$ emissivities based explicitly on transported relativistic electrons, the absence of a significant electron population in the background makes this factor immaterial. Fig. 3a and 3b show with an inverse gray scale the $1.4 \mathrm{GHz}$ spectral index distributions, $\alpha=-\partial \log j_{\nu} /\left.\partial \log \nu\right|_{1.4}=(q-3) / 2,\left(\alpha_{1}\right.$ and $\left.\alpha_{2}\right)$ over the same emissivity range in Models 1 and 2.

Fig. 2 compares emissivities produced by the two "adiabatic" models represented by $j_{1}$ and $j_{2}$. to the "pseudo emissivity", $j_{c}$. The three emissivity images have clear similarities. All show 
a dominant "hot spot", down stream (to the right) of the jet terminal shock. Each produces qualitatively similar patterns of bright emission within the back-flow. The jet is illuminated in the $j_{c}$ model emissivity with patterns that resemble those of the $j_{1}$ model. Of course, the jet is largely invisible in $j_{2}$, because the jet electron population is assumed very small in that model. The model congruencies reflect the strong roles of two key ingredients to the emissivity; namely, the strength of the magnetic field and adiabatic compression and expansion. Note, however, that $j_{1}$ and $j_{2}$ have much greater local contrast than $j_{c}$. Since the magnetic field distributions are identical for all three emissivity calculations, differences in the effective radiating electron distributions must be responsible. That is apparent by noting within smooth flows that $n_{i} \propto \rho^{\frac{q_{i}}{3}}$, so $j_{1,2} \propto n_{i} B^{\frac{q_{i}-1}{2}} \propto \rho^{\frac{q_{i}}{3}} B^{\frac{q_{i}-1}{2}}$. This is actually rather similar to $j_{c} \propto \rho^{5 / 3} B^{3 / 2}$, except for variations modeled in $b_{i}=n_{i} / \rho$ and $q_{i}$, but not modeled by $j_{c}$. The formula given for $j_{c}$ is based on an assumed $\alpha=0.5$, so contrast could be increased in $j_{c}$ by assuming a significantly steeper index. The natural extension of $j_{c}$ to general $\alpha$ would be $j_{c} \propto p^{2 \alpha} \rho^{1-2 \alpha}(B \sin \psi)^{\alpha+1} \nu^{-\alpha}$ (D. Clarke, private communication). For $\alpha=1$ this gives $j_{c} \propto(P B)^{2} / \rho$, just as for $j_{1,2}$ with $\alpha=1$. That would not represent the same effect noticeable in $j_{1}$ and $j_{2}$, however, since from Figs. 2, 3 and 5, we can see that large $j_{1,2}$ come primarily from regions where $\alpha<0.7$.

\subsection{2. "Synchrotron Aged" Model 3}

In Fig. 4 we show the analogous simulated synchrotron emissivity and spectral information for our Model 3, which includes the effects of synchrotron aging . Recall that, except for this influence, Model 3 is identical to Model 1. Thus, Fig. 4a $\left(j_{3}\right)$ can be compared to Fig. 2a $\left(j_{1}\right)$, while Fig. 4b $\left(\alpha_{3}\right)$ can be compared to Fig. 3a $\left(\alpha_{1}\right)$. In addition, we illustrate the level of spectral curvature of Model 3 in Fig. 4c, using of the difference between the simulated spectral indices at $1.4 \mathrm{GHz}$ and $5.0 \mathrm{GHz}\left(\delta_{3}=\alpha_{3}(5 G H z)-\alpha_{3}(1.4 G H z)\right)$. Note that we use formal definitions of $\alpha$ rather than the traditional observed value defined by the ratio of fluxes measured at two distinct frequencies. The intrinsic "observed" index can be found by the simple formula $\alpha_{5}^{1.4}=\alpha+0.5 * \delta_{3}$. These "observed" indices would be almost the same as $\alpha$ near the origin of the jet and in flows just either side of the terminal shock in Fig. 4. On the other hand the "observed" spectra would tend to be $\sim 0.05$ steeper in mid regions of the jet and in most of the back flow, cocoon regions bright enough to show in this figure. But, we mention another caveat with regard to comparisons with directly observed properties. Beyond other complications already mention, including differences between emissivity and surface brightness, a real observation would likely smooth over a physically significant range of sight lines. Non-black regions in Fig. 4 cover a wider range of emissivity values (namely a factor $3 \times 10^{4}$ ) than in Figs. 3 and 4 , since the inclusion of synchrotron aging adds considerable contrast to the emissivity distribution. This factor 10 extension captures approximately the same physical regions as for the other models.

The emissivity distribution, $j_{3}$, for Model 3 including synchrotron aging, is shown in Fig. 4a. As mentioned earlier, the dynamical range found in $j_{3}$ is greater than for any of the other 
models, since there is considerable steepening visible in the spectral index as one moves away from electron sources; namely, the jet origin and strong shocks. Steepening due to aging is apparent even within the jet, where $\alpha_{3}$ increases from 0.7 at the origin to values near 1 even before the first oblique shocks are encountered. Although the emissivity distribution within the jet in Fig. 4a is very similar in appearance to that for Model 1 in Fig. 2a, the factor 10 increase in dynamic range covered in Fig. 2a slightly de-emphasizes the stronger emissivity decreases caused by synchrotron aging. By contrast to the adiabatic models, where the synchrotron spectral index remains near $\alpha \approx 0.7$ almost everywhere in the jet, the oblique jet shocks do produce a noticeable flattening in the spectra of Model 3. The oblique shocks have compression ratios, $\sigma \lesssim 3$ corresponding to a postshock index, $q>4.5 ; \alpha>0.75$, so they only flatten an incident spectrum that has been steepened by radiative losses. In fact, from Fig. 4c it is apparent that in Model $\mathbf{3}$ there is significant spectral curvature in the synchrotron spectrum even within the jet. The nature of that is also visible in the electron momentum distribution plot in Fig. 7c. While the oblique jet shocks are relatively ineffective as electron accelerators, places where these oblique shocks intersect form strong shocks with their normals aligned to the jet axis. They produce spectra synchrotron spectra as flat as $\alpha \sim 0.52$ (see Fig. 5). But, as already mentioned, the portion of the jet flow intercepted by these shocks is very small, so in these simulations they have little impact on the large scale synchrotron emission within these flows.

\subsubsection{General Comments - Electron Transport \& RG Dynamics}

We begin this section with some observations about patterns in the synchrotron emissivity spectra within the jet structures themselves. In Fig. 3 we see that the jet spectra for the adiabatic Model 1 and $\mathbf{2}$ are almost everywhere $\alpha=0.7$, which was the injected spectrum. That is despite the existence of several jet shocks and apparent "knots" in the flow before the terminal shock, where the spectrum flattens to $\alpha \approx 0.54$. Should we have expected to see spectral flattening at the jet "knots"? In these two models the answer is no, since shocks only flatten an incident electron spectrum if it is steeper than the index $q_{s}=3 \sigma /(\sigma-1)$, where $\sigma$ is the compression ratio through the shock (see $\S \S 2.2$ and A.3.2). Thus, only shocks with compression ratios, $\sigma>3.14$ would lead to flattening within these jets. In fact, except very near the jet axis the jet shocks are oblique, with compression ratios, $\sigma<3$, in these simulations. There is one very restricted flatter spectrum region in the jet in Models 1 and $\mathbf{2}$ right on the axis and just to the left of the terminal shock (Fig. 3). There a very strong shock has formed at the intersection of oblique shocks. However, as mentioned earlier, the volume of jet plasma involved is very small, so this feature is hardly noticeable. On the other hand, synchrotron cooling in Model 3 steepens the incident electron spectra coming into the "knots", so that in Fig. 4b we can see clear evidence for spectral flattening at the jet "knots", albeit to indices, $\alpha \gtrsim 0.7$, in these simulations. The details of such features will probably vary with the details of the assumed jet structures and Mach numbers. For example, if the jet flows and magnetic fields conspired to produce the greatest emissivities close to the jet axis, then spectral changes at shocks would be more apparent, since jet shocks do tend to be transverse on axis. 
The observational evidence bearing on this is still limited. Our choice of $q_{o}=4.4\left(\alpha_{o}=0.7\right)$ was intended to represent a "typical" FR II RG jet spectrum, of course. It is most often hard to isolate structures in RGs that are clearly "only" jet flows (see, e.g., Rudnick \& Katz-Stone 1996) because jets are often relatively weak emitters and because they can, therefore, be confused by contamination from the cocoon surrounding them. This makes it especially hard to isolate changes from the jets to the knots, because the contamination from cocoon emission (expected to be relatively steep-spectrum) is greater for the inter-knot regions than in the knots. In some cases where the jet data look convincing, such as for M87 (actually a FR I source), the radio spectrum seems to be virtually constant along the entire jet with no indication of curvature (e.g., Biretta et al. 1991; Meisenheimer, Röser \& Schlötelburg 1996). The extension of this spectrum to the visual band is what strongly constrains aging in M87, since the estimated cooling times for radio emitting electrons are longer than typical estimates of propagation times. And, indeed, there are indications, despite the constancy of $\alpha$ in the radio band, that the radio-to-optical spectrum steepens between the "knots" in the M87 jet (Sparks, Biretta \& Macchetto 1996). In Cyg A the jet radio spectrum appears also to be locally nearly straight, but to exhibit different slopes at different locations (Rudnick \& Katz-Stone 1996; Katz-Stone et al. 1993). Other radio jets may show evidence of steepening of the spectrum along the jet (e.g., Mack et al. 1998), but data do not yet allow detailed analysis in terms of electron transport. Clarke et al. 1986 reported evidence for spectral flattening at a knot in the jet of Cen $\mathrm{A}$. The spectral index flattens to $\alpha \approx 0.7$, from the surrounding emission where $\alpha \approx 1.5$. If these changes really are within the jet flow and not influenced by contamination, this case could easily be accounted for by the effects we see in our simulations.

The spectral index distributions in Fig. 3 show for our adiabatic models that much of the bright emission outside the jet itself, especially in the hot spot, is associated with spectra flatter than the $\alpha=0.7$ expected from the in-flowing jet plasma. Even the brightest parts of the cocoon are dominated by spectral indices with $\alpha \lesssim 0.7$. That must be largely due to acceleration in the terminal shock, of course. Closer examination reveals considerable fine structure, however, in the spectra. Fig. 5 shows, especially for Model 2, that reasonably bright emission is produced with spectral indices ranging roughly over a wide range, $\alpha \sim 0.54-0.75$. The $\alpha \approx 0.54$ emission comes from electrons accelerated in the strong, plane portions of the terminal shock. Near the head of the jet in this model the brightest emission in the back-flow still tends to have relatively flat spectra, $\alpha \lesssim 0.6$. However, it is interesting to note that the spectra of the brighter areas in Models 1 and $\mathbf{2}$ are often steeper farther back in the cocoon. That does not come from radiative losses, which are negligible in these two models, but from increased mixing between flat and steep electron populations. This is an important effect to recognize, since it mimics what is usually assumed to be due to synchrotron aging in the back flow. The specific locations of flat and steep spectra in these examples, should be viewed with caution, of course, since once again we are observing axis-symmetric flows that do not mix in the same ways as the more general 3-D flows will do.

Where do these steep-spectrum electrons come from in the adiabatic models? The steeper 
spectrum emission in the hot spot and the cocoon represents plasma that has escaped passage through the strong, plane terminal shock. One such path is plainly visible for Model $\mathbf{1}$ in Fig. 3a. There we can trace emission with $\alpha=0.7$ all the way from the jet origin on the left into the cocoon back-flow. There are even $\alpha=0.7$ "fingers" of unmodified jet electron populations just outside the jet and near the left of the grid. The source of these electrons is made apparent by the invisibly low $j_{2}$ values (Fig. $2 \mathrm{~b}$ ) in the same regions. That plasma flows into the cocoon through the outer, oblique portion of the terminal shock where the shock compression $\sigma<3$, so the spectrum is unmodified from its original form in the absence of synchrotron aging. We note in an axis-symmetric flow that plasma emerging from the jet terminus near the outer radius of the jet must initially flow back into the cocoon closer to the jet channel than plasma emerging along the jet axis, since 2D flow "streamlines" do not cross. That also explains why near the hot spot flatter spectrum emission tends to extend along the outer, forward parts of the cocoon in Models 1 and 2, since that represents plasma passing through a strong terminal shock nearer the jet axis. As noted, turbulence within the back-flow eventually mixes these populations together, steepening the average spectrum. There is also a related very important source of steep spectrum electrons in the cocoon in these adiabatic models; i.e., there are time periods with little or no perpendicular terminal shock. Then most or all of the emergent jet plasma carries electron populations unflattened by the terminal shock. This is especially relevant when there is fresh electron injection at shocks, as in Model 2. There, the steeper spectrum emissivities can be introduced by electron injection in oblique portions of the terminal shock.

Finally, in the context of the adiabatic models we mention that there is significant, bright emission in Model 2 coming from electrons in the shocked, ambient IGM; i.e., where $C_{j}=0$, so $j_{c}=0$. This comes from IGM electrons injected to the relativistic population by the jet bow shock and now embedded in regions with moderately strong magnetic fields. Fig. 3b shows that the spectral range is large in these regions, with $\alpha \gtrsim 0.6$.

At first glance the emissivity distribution for the synchrotron aged Model $\mathbf{3}$ in Fig. 4a is very similar to the adiabatic models, especially Model 1, which shares the same electron injection history. Even accounting for the greater dynamic range displayed in $j_{3}$ this is especially true near the terminal hot spot. Within the hot spot that similarity extends to the spectra, which are relatively flat in both models. But, closer examination reveals some interesting differences that come from the effects of strong synchrotron aging. First, even within the hot spot, Model 3 displays a substantial spectral range, from close to $\alpha \sim 0.5$ to values greater than $\alpha \sim 1$. In fact, the brightest regions of the hot spot have spectral indices just greater than $\alpha=0.7$, by contrast to values near 0.54 for Models 1 and 2 (see Fig. 5). The strong spectral steepening that takes place at the very front of the terminal hot spot region is a product of the very strong magnetic field that forms at the nose of the emerging jet. This is evident in Fig. 4e and 4f, where it is apparent that the emission from the strongest fields (which lie in this region), while very bright, is also quite steep. From Fig. 5 we can see that the strongest fields in the hot spot are $\sim 8$ times greater than $B_{\text {background, }}$, so from eq. [3-1], the cooling times of the emitting electrons are quite short; namely, 
$\tau_{s} \sim 6 \times 10^{-2} r_{j} / c_{a}$. In that situation, with the absence of any additional acceleration within the postshock flow, spectral steepening is a virtual certainty. In fact, strong spectral index gradients in some RG hot spots have been identified using "spectral tomography" techniques Treichel \& Rudnick 1998), showing that real hot spots have structures that may eventually enable us to explore electron radiative histories and origins, once we understand the behaviors of more realistic models.

For the most part the brighter regions in the cocoon of Model 3 have spectra with $\alpha \gtrsim 1$, and on the whole, the cocoon has a steeper spectrum than the hot spot. These properties are in rough, general agreement with observational expectations (e.g., Dennett-Thorpe et al. 1997; Mack et al. 1998). It is interesting to note that there are "pockets" of flat spectrum emission in the back flow, however. There is a particularly prominent region in the forward-most swirl just above the terminal hot spot, visible in Fig 5. Electrons in these regions were subjected to strong shock acceleration, but were shed in a vortex event without ever passing through a region of strong magnetic field capable of causing strong aging. The field in their current position is actually below $B_{\text {background }}$ (see Fig. 1b), so their cooling time has become a large fraction of the full simulation time. These regions are moderately bright because the electron density is relatively high. As possible evidence that such structures might form in real RGs we mention that Mack et al. 1998 point to the existence of "flat spectrum islands" $(\alpha \sim 0.6)$ in the lobes of NGC 315 where $\alpha \sim 1.3$.

The presence of flatter spectrum populations within the back flow is also related to the fact that additional steepening of the spectrum of the cocoon is slight in the brightest regions in Model 3 once one looks past the most forward portions (see Fig. 4b). From Fig. 1b and Fig. 5f it is plain that the magnetic fields in the visible regions farther back in the cocoon are roughly comparable to those in the forward cocoon or the much of the terminal hot spot. In particular they are greater than $B_{\text {background }}$. Thus, the nominal cooling times based on current conditions would be $\tau_{s}<4 r_{j} / c_{a}$, while the age of the jet at this time is $t \approx 10.67 r_{j} / c_{a}$. The reason for this apparent contradiction between short cooling times and slow aging is the highly unsteady and nonuniform nature of the cocoon flow, especially the magnetic field, in the cocoon. Because of that the electrons spend only a fraction of their lives in strong field regions where they emit strongly. Thus, they cool much more slowly than one would naively predict, based on emissivities. This supports previous suggestions that the radiative ages of RGs appear less than their dynamical ages because of small magnetic field filling factors (e.g., Myers \& Spangler 1985) or filamentary magnetic field structures (e.g., Eilek et al. 1997).

We can recognize some additional and generally interesting behaviors from Fig. 5. For example, from Fig. 5a and $5 \mathrm{c}$ for most of the points representing shocked jet emission (including all the points with $\alpha<0.7$ in Fig. 5a) there is an envelop restricting the strongest emission to the flattest spectra. Note, for example, in Model 1 none of the brightest emission outside the jet comes from regions with $\alpha>0.6$, despite the fact that the magnetic fields in the fainter, steep spectrum emitting regions are as strong as in the bright, flat spectrum regions (see Fig. 5b). The "nose" of the $\left(j_{\nu}, \alpha\right)$ distribution in Fig. 5a, 5c, 5e corresponds to the terminal hot spot 
emission, where fields are strong and electron densities are high. The largest emissivities naturally correspond to the strongest field regions. The converse is not true, however; strong fields do not necessarily produce a large emissivity. The other ingredient in $j_{\nu}$, of course, is the electron density, $f(p)$. Fig. 5 is, therefore, showing us a wide range of electron densities associated with magnetic field strengths contributing to the emission, and even some degree of anti-correlation between field and $n_{i}$ in the back-flow. Back-flowing plasma has mostly undergone adiabatic expansion after emerging from the jet, while the $B_{\phi}$ component of the magnetic field has been enhanced in some locations by stretching of field lines at the same time. In Model 3 (represented in Fig. 5e,f), where synchrotron aging also acts to reduce emission by steepening the spectrum, the patterns are actually very similar to those in the adiabatic models. However, much of the emission is shifted upward to steeper spectra; roughly by $\Delta \alpha \sim 0.2$. The points remaining near $\alpha \sim 0.5$ correspond to shocked points within the jet near its axis, and points just past the jet terminal shock, where aging effects are not yet important. Finally, we comment that more generally, there are many "micro patterns" visible in Fig. 5. Those tend to trace hydrodynamical features, so that they are revealing histories of the electrons. Unfortunately, those flow patterns are usually unsteady so that it would be difficult, if not impossible to use them to determine explicit connections between individual points and flow dynamics.

\section{Conclusions}

We have developed a simple but effective numerical scheme for transport of low energy relativistic electrons suitable for studies in time dependent simulations of radio galaxies. The scheme can follow the evolution of electrons accelerated by first order Fermi acceleration in shocks, second order Fermi acceleration, adiabatic cooling and synchrotron cooling, a.k.a. "aging", in smooth flows. From the electron spatial and momentum distribution and the magnetic field properties found through the fluid evolution one can directly compute the radio synchrotron emission. In this paper we have applied those methods to axis-symmetric MHD jet flows including first order Fermi shock acceleration and synchrotron aging effects on the electrons, in order to identify some of the behaviors that may be expected in multi-dimensional flows. We begin with $2 \mathrm{D}$ flows since they are much simpler than 3D flows to study and to understand. We have focussed our discussion on effects that should occur in both 2D and 3D flows, so that what we learn here will facilitate future studies with more general flows. Briefly, the most important findings of this work that we believe to be independent of our specific simulation assumptions are:

- The fact that jet terminal shocks are not simple, steady plane structures should play a major role in determining the properties of synchrotron emission within the terminal hot spot and in the lobes generated by the jet back flow. In fact, the outflows are inherently complex, because of the basic "driven" character of a jet flow. As pointed out recently by Sato et al. 1996, Complexity is basic to all driven non-equilibrium plasma flows. RGs are dramatic examples of such flows, so they should be influenced by episodic patterns of instability and reorganization. In 
particular, the strength and form of the terminal shock is constantly and sometimes dramatically changing. Thus, the nonthermal electron population emerging from the jet may encounter a wide range of shock types and strengths, as well as magnetic field environments.

- Because of the previously mentioned behaviors, we may expect to find a complex range in synchrotron spectral and brightness patterns associated with terminal hot spots and lobes. These include the possibility of steep spectral gradients (of either sign) within hot spots, the potential in lobes for islands of flat spectrum electrons within steeper spectral regions (or the reverse) and spectral gradients that result from the dynamical history of a given flow element rather than from synchrotron aging of the embedded electrons.

- Synchrotron "aging" in the lobes tends to proceed more slowly than one would estimate from regions of high emissivity. That is a consequence of the fact that those regions are generally places where the magnetic fields are the strongest, so that the instantaneous rates of energy loss are atypical of the entire history of the electron population. This feature is apparent even in axis-symmetric flows; it should be stronger in real, 3D flows, since for equivalent initial structures, local magnetic field enhancements should be greater in 3D (Jones, Ryu \& Frank 1998). Our finding supports earlier suggestions that nonuniform field structures may help to explain why dynamical ages of FRII sources often seem to be greater than the apparent age of the electrons radiating in the lobes (e.g., Eilek et al. 1997).

There are many uncertainties inherent in attempts to model such complex objects as RGs. The flows are inherently intricate and many key parameters are largely unknown at present. That is why we have examined first the simplest systems that may be qualitatively representative and have tried to restrict our questions to those not wholly dependent on uncertainties coming from the limitations imposed by our various choices. Even from these few initial results we can see the importance of modeling electron acceleration and transport and the significance of shock history to the radio spectra in RG hot spots and lobes. The interpretation of synchrotron spectra in RGs clearly is not simple, but represents the combined influence of several dynamically coupled factors. That should, perhaps point us to another analysis approach; namely, thinking directly in terms of "Complexity" itself. Sato et al. 1996 argue that in such systems we need to shift our paradigm from a focus on specific elements (e.g., the existence of a terminal shock that can accelerate particles) to the consequences of interactions among elements (e.g., long range order and the relationships between dynamical structures). The task then becomes one of understanding how the "microphysics" (e.g., particle transport) can help to establish relationships among the various system elements.

Support for this work was provided by NSF grants AST93-18959, INT95-11654 and AST9616964, by NASA grant NAG5-5055 and the University of Minnesota Supercomputing Institute. The work by DR was supported in part by KOSEF through the 1997 Korea-US Cooperative Science Program 975-0200-006-2. We gratefully acknowledge helpful discussions with B.I. Jun and especially Hyesung Kang during development of the electron transport scheme and with Larry 
Rudnick about practical connections between simulations and radio galaxies. We also thank the referee, David Clarke, for very helpful comments and discussion on the manuscript.

\section{A. Appendix}

\section{A.1. Constraints on Computational Diffusive Electron Transport in RGs}

A number of studies have considered time dependent solutions to eq [2-1] for charged particles in shocked flows (e.g., Falle \& Giddings 1987; Drury 1991; Kang \& Jones 1991; Kang, Jones \& Ryu 1992; Berezhko, Ksenofontov \& Yelshin 1995; Kang \& Jones 1995; Kang \& Jones 1997). Those studies and most others like them have focussed on the transport of nonthermal ions. Although diffusive transport is distinct for nonrelativistic electrons (e.g., Levinson 1996), relativistic electrons can be treated exactly like ions of similar rigidity, so the above treatments are directly relevant to the transport of electrons responsible for synchrotron emission. All of the papers mentioned, however, considered 1-D flows for a single shock system. This limited application reflects a severe numerical constraint in solving eq. [2-1], especially for low energy particles. With existing numerical schemes it is not practical to carry out a full and direct solution to eq. 2-1 within a "large-scale", complex multi-dimensional flow. The reasons are straightforward. First, note that eq. 2-1] applies only outside of shocks, since its validity is restricted to particles whose scattering lengths and gyroradii are large compared to the shock thickness, which should be a few thermal ion gyroradii (e.g., Kang \& Jones 1995; Kang \& Jones 1997 and references therein). Its application to diffusive shock acceleration depends entirely on being able to match upstream and downstream solutions properly at the shock (e.g., Drury 1983; Drury 1991). An accurate numerical solution to eq. 2-1] requires that the shock be a discontinuity or close to it. Numerical schemes for compressible fluid dynamics, including the one we employ, spread shock structures over at least one or two zones. One of the necessary matching conditions for diffusive electron transport is that the distribution $f$ be continuous across the discontinuous physical shock. So, if we apply eq. [2-1] at a shock, we must demand, to avoid errors within the (unphysical) numerical shock, that the advective flux term in eq. 2-1] integrate to the same value as at a true discontinuity. It is simple to show that the errors in this are first order in $\Delta x_{s} / x_{d}$, where $\Delta x_{s} \geq \Delta x$ is the numerical shock thickness, $\Delta x$ is a zone width at the shock and $x_{d}=\kappa / u_{s}$ is the diffusion length for energetic particles at the shock, with $u_{s}$ being the velocity jump across the shock. Therefore, we must have $\Delta x_{s} / x_{d}<<1$.

To see what constraint this gives in a RG application we need to estimate $x_{d}$. There is empirical evidence that near shocks the diffusion coefficient, $\kappa$, is a steeply increasing function of momentum with a form resembling the so-called Bohm diffusion coefficient, $\kappa \sim r_{g} w$, where $w$ is the particle speed (e.g., Ellison et al. 1993). For GeV electrons in a magnetic field exceeding $\sim 10 \mu \mathrm{G}$, for example, we would estimate for Bohm diffusion near a strong shock with speed, $u_{s} \sim 10^{8} \mathrm{~cm} \mathrm{~s}^{-1}$, that $\kappa \lesssim 10^{22} \mathrm{~cm}^{2} \mathrm{~s}^{-1}$ and $x_{d} \lesssim 10^{14} \mathrm{~cm}$. Lower energy electrons, especially those 
near shock injection energy, would give smaller $x_{d}$ by at least two orders of magnitude. Thus, a direct simulation in a $\mathrm{RG}$ of the full eq. [2-1] for electrons even at $\mathrm{GeV}$ energies would require numerical shocks much thinner than $10^{14} \mathrm{~cm}$. To include electrons down to injection energies in this situation we would necessarily require numerical shocks to be no thicker than about an AU. This is a spatial constraint, so temporal sub-cycling techniques to deal with associated rapid acceleration are not sufficient to deal with this problem. Since the simulation scale in a RG is typically tens or hundreds of kpc one would need to resolve shocks on a scale no more than about $10^{-6}$ of the main grid scale and much smaller than this to capture the particle behavior with eq. [2-1] near injection energies. For simple, 1-D flows this kind of refinement near shocks may be feasible using a nonuniform grid, although one still must deal with numerical issues coming from the accompanying orders of magnitude range in the time steps required for the solution. An adaptive mesh refinement (AMR) approach may eventually make the analogous multi-dimensional treatment feasible, but until those codes are available for MHD, including diffusive transport, an alternative way around the problem is necessary for meaningful calculations.

\section{A.2. A Practical Approach to Dealing with Electron Transport}

Fortunately, for the problem at hand we can actually exploit the small diffusion length constraint to introduce a simplified version of eq. 2-1] that still correctly captures the essential physics of electron transport, at least when the dynamical feedback of the electrons can be ignored; that is when the electrons can be seen as "test particles." Jones \& Kang 1993 applied a very simple version of such a scheme several years ago, and Jun \& Jones 1998 have briefly described a variant of the one discussed below. To begin, we remind readers of the point in $\S A .1$ that we should distinguish between behaviors at shocks and within smooth flows, away from shocks. Therefore, we divide discussion of our proposed scheme into these topics and begin with shocks, since the emergent properties of the electron distribution help to determine our approach in smooth flows.

\section{A.2.1. Transport Through Shocks}

At shocks we must properly balance the change in advection at the shock against diffusion away from it. From the arguments in the preceding section, we must find a way to make the numerical shock much thinner than a conventional numerical zone width. The most obvious numerical solution to this problem is to treat the shock as a real discontinuity; i.e., confine the shock transition to a zone boundary as far as particle acceleration is concerned. Generally, as mentioned earlier, that involves imposing the matching conditions to $f$ across the shock (e.g., Drury 1983; Drury 1991) to properly account for both diffusion and advection on both sides of the shock. Drury 1991 used this method to find analytic time dependent test particle solutions for $f$ at a plane shock, for example. Berezhko, Ksenofontov \& Yelshin 1995 used it numerically to treat time dependent ion acceleration at the spherical blast wave of a supernova 
remnant, including backreaction on the local flow. Those particular methods are not practical to apply to numerical schemes in general flow patterns, because they are computationally intensive and require highly customized assumptions. However, for the low energy electrons in RGs that are of interest to us in this paper, it is possible to accomplish the required outcome in a manner that is easily adapted to general numerical schemes. The key points are: 1) as already discussed, diffusion lengths are very small compared to typical numerical zones on either side of the shock for the particles under consideration; i.e., $\Delta x>>x_{d}$; and 2) the characteristic diffusion timescale, $t_{d}=x_{d} / u_{s}$ adjacent to the shock is much smaller than numerical time steps associated with the standard CFL condition; i.e., $\Delta t \sim \Delta x / u_{s}>>t_{d}$. In fact, $\Delta t / t_{d} \sim \Delta x / x_{d}$, so, from our earlier discussion, both conditions are easily satisfied by many orders of magnitude in RG simulations.

The first point means that the entire upstream "precursor" to the shock that controls evolution of $f$ is well-contained within a single numerical zone. This prohibits treatment of backreaction by the nonthermal particles on the flow, since that depends on knowing the velocity gradient within the precursor. But, electrons accelerated in shocks are generally thought not to produce significant backreaction (e.g., Blandford \& Eichler 1987). In the work we present here the nonthermal electron population is not dynamically coupled back to the flow. The second point also means that the precursor is formed instantaneously by comparison to the numerical, dynamical time step. More directly, it means that the timescale for particles to be accelerated at the shock to the energies of interest is also very small compared to the numerical time step. Assuming for simplicity that $\kappa$ is the same upstream and downstream of a strong shock, the mean time for particles to be accelerated from an initial momentum, $p_{o}$, to momentum, $p$, can be written approximately as $t_{a} \approx \frac{20}{u_{s}^{2}} \int_{p_{o}}^{p} \kappa\left(p^{\prime}\right) d \ln p^{\prime}$ (Lagage \& Cersarsky 1983). For Bohm diffusion of relativistic electrons near a strong shock $t_{a} \sim 20 \times t_{d} \sim 2 \times 10^{7} E_{\mathrm{GeV}} /\left(B_{10} u_{s 8}^{2}\right)$ sec, where $u_{s 8}$ is the shock speed expressed in units of $10^{8} \mathrm{~cm} \mathrm{~s}^{-1}$. For $\mathrm{GeV}$ electrons this is about a year for our selected characteristic numbers, compared to a typical CFL-determined dynamical time step in a RG simulation $\gtrsim 10^{3}$ years. The synchrotron radiative cooling, or "aging" timescale, $t_{s} \sim 10^{15} /\left(\nu_{9}^{\frac{1}{2}} B_{10}^{\frac{3}{2}}\right)$ sec, should be comfortably longer than either of these for the particles of immediate focus, so we can deal with effects of radiative cooling separately on large scales. It has been demonstrated analytically (Drury 1991) and numerically (Kang \& Jones 1991) that after a time $\Delta t$ a particle distribution $f_{o}$ flowing into a steady plane shock emerges downstream with the appropriate steady state solution for $p / p_{a t}<<1$, where $p_{a t}$ is the momentum that can be reached by diffusive acceleration during the time interval $\Delta t$, as defined by $t_{a}$. For our application, where $p_{a t}(\Delta t)$ is much larger than momenta of interest if $\Delta t$ is a typical CFL hydrodynamical time step, it becomes unnecessary to treat the detailed time evolution of $f$ at shocks with eq. [2-1]. Instead for $\mathrm{GeV}$ electrons we can assume immediately downstream of a shock that in response to diffusive shock acceleration $f \propto p^{-q}$, where $q=\min \left(q_{s}, q_{o}\right)$, with $q_{o}=-\partial \ln f_{o} / \partial \ln p$ the logarithmic slope of the in-flowing electron distribution at momentum $p$, and $q_{s}=3 r /(r-1)$, the standard diffusive shock acceleration power law slope. In this $r=\rho_{2} / \rho_{1}$, the compression ratio through the shock (e.g., Drury 1983). 
The above discussion also leads to the conclusion that, at least as it emerges from shocks, the electron momentum distribution for energies of direct relevance for radio emission will generally be well-approximated by a power law. That emergent form may change in subsequent propagation over finite lengths due to radiative losses, mixing between particle populations with different initial spectra or second-order Fermi acceleration. Nonetheless, this suggests an obvious simplification to the energy distribution that greatly reduces the effort required to solve eq. [2-1] outside of shocks in RG flows. In particular $q=-\partial \ln f_{o} / \partial \ln p$ should be a slowly varying function of $\ln p$. Then, for numerical purposes it makes sense to approximate the momentum distribution as a piece-wise power law over bins of finite width in $\ln p$. The practical coarseness allowed for such a momentum grid will be determined by the expected curvature of the spectrum and the information needed from the spectrum in the momentum range of particular interest.

\section{A.2.2. Transport in Smooth RG Flows}

In smooth flows, eq. [2-1] is directly applicable. Here the relative importance of advective and diffusive fluxes is given by a ratio $u \delta x / \kappa$, where $u$ is a characteristic advection speed across zone boundaries and $\delta x$ is the scale length of $\nabla f$ in the smooth flow. Assuming $\delta x>\Delta x$, it is easy to show with the diffusive properties mentioned earlier, that on a typical numerical grid with $\sim 10^{3}$ zones spanning the RG dimensions in any direction, $u \delta x / \kappa>u \Delta x / \kappa>>1$. Thus, advection of $f$ for low energy particles in the smooth flows should always dominate over spatial diffusion even if the spatial diffusion coefficient is several orders of magnitude greater than that we discussed next to the shocks. We conclude that we can neglect the spatial diffusion term in eq. 2-1 for low energy electrons in RGs except at shocks.

\section{A.3. A Simple Electron Transport Scheme for RGs}

To solve eq. 2-1] we should divide a momentum range of interest into $\mathrm{N}$ bins bounded by $p_{0}, \ldots, p_{N}$. It is most practical to work with logarithmic intervals in $p$, so we generally use $y_{i}=\ln p_{i} / p_{0}$, giving bin widths, $\Delta y_{i}=y_{i+1}-y_{i}=\ln p_{i+1} / p_{i}$. For a conventional, finite difference treatment of eq. [2-1] it is necessary to use bins with $\Delta y_{i}<<1$ (e.g., Kang \& Jones 1991). However, with the conservative, finite volume approach defined here, it is practical to use much greater bin widths, $\Delta y_{i} \gtrsim 1$, when the smooth behavior of $\ln f(p)$ outlined in $\S$ A.2 applies.

\section{A.3.1. Smooth Flows}

To begin, we can integrate equation [2-1] within each momentum bin to define $n_{i}=4 \pi \int_{p_{i}}^{p_{i+1}} p^{3} f d \ln p$ as the number of electrons in the bin. It is convenient (but not necessary) to normalize $n_{i}$ by the total plasma mass density, to form $b_{i}=n_{i} / \rho$. Then eq. [2-1] 
becomes, for one spatial dimension the conservative, finite volume equation,

$$
\frac{d b_{i}}{d t}=\left.4 \pi\left(\frac{1}{3} \frac{\partial u}{\partial x}+\frac{D}{p^{2}} \frac{\partial \ln f}{\partial y}\right) \frac{p^{3} f}{\rho}\right|_{p_{i}} ^{p_{i+1}}+\frac{\partial}{\partial x}\left(\left\langle\kappa_{i}\right\rangle \frac{\partial b_{i}}{\partial x}\right)+\frac{Q^{\prime}}{\rho},
$$

where $\left\langle\kappa>=\frac{\int p^{2} \kappa \nabla f d p}{\int p^{2} \nabla f d p}, Q^{\prime}=4 \pi \int_{p_{i}}^{p_{i+1}} p^{2} Q d p\right.$, and $d / d t$ is the Lagrangian time derivative. No approximations have been added to obtain eq. A1] from eq. 2-1.

Now we explicitly include the two simplifying features into eq. A1 that enable us to treat low energy electron transport efficiently in RGs. We shall also define $Q^{\prime}$ specifically, so that it accounts for synchrotron losses and shock injection of low energy electrons. Remember that eq. [2-1] or A1] applies only to the smooth flows. In those regions we assume the piecewise power law $f(p)=f_{i}\left(p / p_{i}\right)^{-q_{i}}$ within $p_{i} \leq p \leq p_{i+1}$, so that

$$
n_{i}=4 \pi \frac{f_{i} p_{i}^{3}}{q_{i}-3}\left[1-\left(\frac{p_{i}}{p_{i+1}}\right)^{q_{i}-3}\right] .
$$

Since $f(p)$ is continuous we can also write $f_{i+1}=f_{i}\left(p_{i} / p_{i+1}\right)^{q_{i}}$. That enables us to find the fluxes at momentum boundaries in eq. A1] to account for adiabatic expansion, second-order Fermi acceleration or radiative cooling. Then also recall from $\S \mathrm{A} .2$ that we can neglect the spatial diffusive transport term in eq. A1 for low energy electrons within smooth portions of flows for the conditions expected in RG simulations.

Synchrotron aging of electrons is straightforward to include in this formulation when there is rapid angular redistribution of electrons (a necessary condition for the validity of eq. [2-1]). Then at momentum $p$ there is a flux $4 \pi p^{2} \dot{p}_{s} f$, with $\dot{p}_{s}=-\frac{p^{2}}{\tau_{s o} \hat{p}}$, where $\tau_{s o}=\frac{3}{4} \frac{(m c)^{2}}{\sigma_{T} U_{B}} \frac{1}{\hat{p}}$ defines the cooling time at some convenient momentum $\hat{p}$ (see, e.g., Jones, O'Dell \& Stein 1974). In this expression, $\sigma_{T}$ is the Thomson cross section, and $U_{B}=B^{2} /(8 \pi)$. To account for inverse Compton cooling from the cosmic microwave background $B^{2}$ should be modified to $B^{2}+B_{\mu}^{2}$, where $B_{\mu}=3.2 \mu \mathrm{G}$ at the current epoch. Putting these features together, we are left in smooth flows to solve the simple transport equation

$$
\frac{d b_{i}}{d t}=\left.4 \pi\left(\frac{1}{3} \frac{\partial u}{\partial x}-\frac{q D}{p^{2}}+\frac{1}{\tau_{s o}} \frac{p}{\hat{p}}\right) \frac{p^{3} f}{\rho}\right|_{p_{i}} ^{p_{i+1}} .
$$

Since eq. [A3] is in conservation form, our main constraint is the accuracy of the fluxes written on the right hand side. Given an initial set of $b_{i}$ and $q_{i}$, along with underlying plasma properties, those can be found accurately at the beginning of the time step, within the piecewise power law approximation by using eq. [A2] and the continuity of $f$. The fluxes can easily be time centered, by using the slope of $f$, to achieve second order time accuracy in the update. One must, of course, include a constraint on the time step, $\Delta t$, beyond the MHD CFL condition. That can be expressed conservatively to account for adiabatic, synchrotron and second-order acceleration effects by the combined condition, $\Delta t<\max \left(\Delta x / a, \tau_{s o} \hat{p} / p_{N}, p^{2} /(q D)_{\min }\right)$, where $a$ is the fastest MHD signal speed. The first condition, for adiabatic expansion, corresponds to the standard CFL condition. The other two need to be examined explicitly, but so long as the synchrotron cooling 
and second-order acceleration take place on "hydrodynamical timescales", they will also usually be enforced by the CFL condition.

In general $q_{i}$ will also change during a time step. That set of values is updated from the new distribution $b_{i}$ by inverting eq. [A2], and again using the continuity of $f$. An appropriate set of boundary conditions is also required, of course. We expect spectral curvature will be common at the highest momenta, but usually minimal at the lowest momenta. So, for the simulations presented here we have assumed that $q_{i}$ is continuous at $p_{0}$, but that the spectral curvature is continuous at $p_{N}$. The solution to $q_{i}$ must be done iteratively. As pointed out by Jun \& Jones 1998, it is possible in principle to find $q_{i}$ exactly if the momentum bins are of uniform size and one applies the boundary condition $q_{o}=q_{1}$. For significantly curved spectra, however, we have found a simple, approximate method to be relatively fast and sufficiently accurate in these applications. In this method we assume for the inversion of eq. [A2] to find $q_{i}$, that $q$ is a linearly varying function of $y$ between the centers of adjacent momentum bins; that is, there is uniform spectral curvature between adjacent bin centers. Thus, $q_{i}$ is interpreted as the average $q$ (or midpoint value) within the interval $\left[y_{i-1}, y_{i+1}\right]$. Then it is straightforward to design a Newton-Raphson iteration scheme to solve for each $q_{i}$. In practice we use only a single iteration from the initial guess

$$
q_{i}(\text { trial })=3-\frac{\ln \frac{b_{i+1}}{b_{i}}}{\Delta y_{i}},
$$

which would be exact for a pure power law on uniform bins.

The number of bins necessary in using eq. A3 over a given momentum range will depend on the amount of spectral curvature expected. Jun \& Jones 1998 showed if one can assume that $f(p)$ is a pure power law at any given physical location, then two momentum bins are sufficient to follow the evolution of $f$ accurately, accounting for adiabatic expansion, advection and consequent mixing of different power laws. In our application that is not adequate, since we may expect significant deviations to develop from pure power law form. On the other hand, from thermal injection energies to those $\sim \mathrm{GeV}$ the relevant $\Delta \ln p<20$, while a range $|\Delta q|=2 \Delta \alpha=3$ would cover electron populations producing synchrotron spectra with slopes $0.5 \leq \alpha \leq 2$, assuming $q \geq 4$. Greater curvature would involve spectral slopes, $q>7$, so that very few particles would be represented at the higher energies. As demonstrated below we have found that it is possible to capture the evolution of electron spectra utilizing only a few momentum bins for practical problems. The computational cost of following each electron momentum bin is roughly comparable to following each dynamical variable in the flow (of which there are eight in MHD). The same statement would apply for a conventional scheme to solve eq. [2-1], such as that we used in Kang \& Jones 1991; but there, a much larger number of momentum bins would be necessary and much finer spatial resolution near shocks would be needed to obtain meaningful solutions in RGs (see $\S \mathrm{A} .1$ and comments at the end of $\S \mathrm{A} .4$ ). 


\section{A.3.2. Shocks}

To model "diffusive shock acceleration", following the arguments of $\S \mathrm{A} .1$, we impose at detected shocks the analytic solution outlined in $\S$ A.2. That is, the emerging distribution has a form $f(p) \propto p^{-q}$, where $q=3 r /(r-1)$, with $r$ the compression ratio through the shock provided $q_{s}<q$, where $q$ is the in-flowing value in a momentum bin. Normalization of $f$ is determined by the total number of electrons; that being the sum of the incident population in the interval $\left[p_{0}, p_{N}\right]$ and any injected electrons as defined below. We ignore shocks with density jumps $r<\frac{7}{4}$ $\left(q_{s}>7 ; \alpha>2\right)$, since they contribute very few nonthermal particles.

For fresh injection at shocks we adopt a commonly used simple model (e.g., Kang \& Jones 1991); namely, we inject a small, fixed fraction, $\epsilon$, of the thermal electron flux through the shock. Thus inside a shock we solve the equation $d b / d t=Q_{i n j}^{\prime} / \rho$, where $b=\sum b_{i}$ and

$$
Q_{i n j}^{\prime}=\frac{\epsilon W_{s}}{\mu_{e} m_{H}}
$$

$W_{s}$ is the Lagrangian shock velocity, $\mu_{e}$ is the electron mean molecular weight, $m_{H}$ is the mass of the proton. As for electrons flowing in from upstream, shock injected electrons are distributed in momentum as a power law with the appropriate slope from diffusive acceleration theory, as described before.

\section{A.4. Tests}

We have carried out numerous tests of the method described above and find it to be both very stable and to produce consistent results, provided roughly $\left|\Delta q_{i, i+1}\right|<0.75$. In the simulations described here we have constrained $\left|\Delta q_{i, i+1}\right|<0.67$. Our various tests included 1-D adiabatic advection of analytically defined convex and concave spectra, as well as shock tube simulations. We carried out a variety of 2-D simulations, such as spherical blast waves and obliquely intersecting shocks, where we could compare analytic expectations of the resulting electron spectra. We also tested the ability of the scheme to handle synchrotron aging by following the evolution of an initial power law electron distribution compared to the well-known analytic result of Kardashev 1962 (but, most commonly associated with Jaffe \& Perola 1973) for synchrotron aging with rapid pitch angle redistribution.

A 2-D application of this scheme to the propagation of a young SNR impacting an interstellar cloud is presented in Jun \& Jones (1998), and can serve to demonstrate its ability to capture expected behaviors correctly for a spherical blast wave, as well as for mixed flows. We offer here another simple 2-D test calculation that could be used by other workers for quantitative comparison. It involves the interaction between two obliquely intersecting plane hydrodynamical shocks, and is illustrated in Fig. 6. Moderate strength shocks are more challenging to capture correctly with regard to electron acceleration, so the example is designed to create several shocks 
with Mach numbers in the range 1.5 - 10. There are five dynamical regions, as outlined below and as shown in Fig. 6a. Region 1 is at rest, with $\rho_{1}=1$ and $P_{1}=0.6$, giving unit sound speed. Region 2 (also uniform) lies behind a Mach 10 right-facing shock initially placed vertically in the middle of the box. The initial conditions in region 2 are, thus, $\rho_{2}=3.884, P_{2}=74.88, u_{x 2}=7.425$, $u_{y 2}=0$. Region 5 similarly lies behind a Mach 4 shock, which, in this case is propagating diagonally up and to the left. This shock was initially placed so that it bisected both boundaries, and, thus, was just touching the vertical shock. Initial conditions in region 5 were, $\rho_{5}=3.368$, $P_{5}=11.85, u_{x 5}=-1.989$ and $u_{y 5}=1.989$.

Propagation of each of the two original shocks into the postshock flows of the other creates new regions 3 and 4, which are separated by a slip line. We carried out this test on a $512 \times 512$ Cartesian grid. Except for the right edge of the box standard continuous boundaries were used. On the right these were modified to include the symmetry of the flow there, thus preserving its self-similarity. The whole flow pattern is self-similar, in fact, except near the bottom of the box, where it is not possible to construct suitable boundary conditions. That leads to the curvature in the shocks visible there, and the incipient vortex at the bottom of the slip line. Using shock polars (e.g., , Courant \& Friedrichs (1976)) it is simple to compute that regions 2 and 3 are separated by a Mach 1.51 shock, while regions 4 and 5 are divided by a shock with Mach number 3.66.

For this particular test there was an initial cosmic-ray electron population that was a uniform $1 \%$ of the thermal electron population in each region. The cosmic-rays arbitrarily were given an initial, uniform power-law form with index, $q=4.5$, corresponding to original injection at a Mach 3 shock. Many other spectral choices could be made, as well. There was no injection of cosmic-ray electrons at the shocks during the simulated flow from this point, although it is straightforward to include that effect, as well.

Fig. 6b illustrates the computed distribution of spectral indices after the flow has evolved. Since the flow is self-similar, except for the mentioned boundary effects, the chosen time does not matter, so long as it is sufficient to allow significant material to pass through each shock. As mentioned the electrons initially had the same spectrum everywhere. Particle acceleration at the shocks during their propagation during the simulation has modified the electron spectra in regions 2', 3", 4' and 5'. The steady-state spectrum behind a Mach 1.51 shock (region 3) from a monoenergetic incident flux would be $q=7.11$. Since that is steeper than the initial spectrum there is no change between regions 2 and 3'. However, region 3" was first processed by the Mach 10 shock, so we find, as expected, electrons there to have the same form as those in region 2'. From the analytical jump conditions we predict the spectral indices in regions 2', 4', and 5' to be respectively, $q_{2^{\prime}}=4.040, q_{4^{\prime}}=4.323, q_{5^{\prime}}=4.267$. In this numerical example they come out to be $q_{2^{\prime}}=4.041, q_{4^{\prime}}=4.333$, and $q_{5^{\prime}}=4.299$, with variations less than or of order $10^{-4}$. Thus, the errors in $q$, which are naturally greatest for oblique shocks on a Cartesian grid, are all less than $1 \%$. Since there is little mixing and no synchrotron aging during this test calculation, all the spectra should very good power laws, which we confirmed using 4 momentum bins covering $\Delta \ln p=20$. 
This test also demonstrates the ability of our routine to advect the electrons cleanly in the different momentum bins, since the spectral indices are computed from the actual particle distributions among the distinct momentum bins. So, for example, the population differences between regions 3' and 3" in the higher momentum bins are more than an order of magnitude, and that difference is maintained accurately with a transition over about three spatial zones. Any error or significant diffusion there would lead to a spurious flattening of the spectrum along the boundary region 3'. The spectra there computed for the two highest momentum bins is almost precisely the same as for the two lowest bins. Similar comments apply to the slip line between regions 3" and 4'. Recall, of course, that we assume for these calculations that real, physical diffusion is very small on scales of the computational zones.

To demonstrate further the behavior of our electron transport scheme we include Fig. 7, which shows a snapshot of the results from three 2D test simulations exactly like the Model 3 jet described in $\S 3$, except that the spatial resolution here was only half as fine. In these calculations an electron population with $p_{0}=\mathrm{mc}, p_{N} \approx 10^{5} \mathrm{mc}$ and a power law index $q=4.4$ enters with the jet on the left of the grid. That population is modified by adiabatic and synchrotron cooling and by any shocks encountered along the way. The grayscale image shows the log of the total electron number near the end of the simulation. For numerical convenience, the computation also includes a small population of steep spectrum $(q=7)$ background electrons in the ambient medium. They are faintly visible in the image, especially through their compression at the jet bow shock. Fig. 7 also shows the computed electron momentum spectral index at four selected locations on the grid. Each plot gives the values of $q_{i}$ found in simulations with the number of momentum bins, $N$ set to 4 (triangles), 8 (stars) and 12 (squares).

Note first the plot (b) in Fig. 7, which shows the spectrum of the background population, changed only in response to synchrotron aging in a uniform background magnetic field. The solid curve gives the prediction, $q(p, t)=-\partial \ln f / \partial \ln p$ from Kardashev 1962; namely,

$$
q(p, t)=q_{0}+\left(q_{0}-4\right) \frac{\frac{t p}{\tau_{s o} \hat{p}}}{1-\frac{t p}{\tau_{s o} \hat{p}}},
$$

where $q_{0}$ is the initial slope. In this case $q_{0}=7$, and the time shown was $t=\tau_{s o}$. Equation A6 is valid, of course, only for $\frac{t p}{\tau_{s o} \hat{p}}<1$, since higher momentum particles have all cooled below $p_{\text {cool }}=\hat{p} \frac{\tau_{s o}}{t}$. In this case $p_{\text {cool }}=\hat{p}=10^{4}$ mc. All three numerical results agree with eq. A6 in the range of momenta where the curvature is less than the numerical imposed constraint mentioned earlier; namely that the change in $q_{i}$ between adjacent bins is less than $\frac{2}{3}$. For $N=4$ violation occurs above $p \sim 10^{3} \mathrm{mc}$ in this case, but for both $N=8$ and $N=12$ the numerical results are in reasonable agreement below about $\frac{1}{3} p_{\text {cool }}$. The deviation at higher momenta has little practical consequence, since the numerical slope there is still so steep and the associated $b_{i}$ so small that it will have little influence on the evolution at lower momenta (vanishing flux contribution) and would contribute negligibly to emission processes.

Similarly with each of the other three particle spectra shown (all corresponding to the 
dynamical time displayed in the image), the agreement between the $N=8$ and $N=12$ solutions is very good until the computed spectra are too steep to influence observable properties. For the two spectra (c) and (d, where curvature is small below $p \sim 10^{4} \mathrm{mc}$, the $N=4$ solution agrees quite well with the other two. We conclude that the scheme is effective and reasonably robust. When there is only modest spectral curvature in the momentum range of interest, as few as 4 momentum bins may be adequate to capture the evolution of the spectrum. For the first two RG models presented in $\S 3$, synchrotron aging is negligible, so we use $N=4$ in those calculations. In situations where strong curvature needs to be captured, such as those involving strong synchrotron aging, a larger number of bins are needed. In the Model 3 presented in this paper we consider emission almost entirely from electrons having $p<10^{4} \mathrm{mc}$, and we judge there that $N=8$ is adequate for the issues under discussion. This ability to capture basic behaviors of electron transport in RG flows with only a few momentum zones results from the conservative nature of the scheme, the quasi-power law properties of the electron distribution and the fact that the microphysics of the electron transport can be analytically well-approximated in smooth flows and at shocks. The impact of this fortuitous outcome is clear when one considers that any scheme to follow the electrons will require a computational effort per variable tracked that is comparable to the effort necessary to follow a dynamical variable, such as the gas density. In this scheme we need $2 N$ variables $\left(b_{i}, q_{i}\right)$, with $N \sim$ a few, so that the net effort to follow the relativistic electrons is comparable to the net MHD effort in the calculation. In our experience, direct solution of eq. [2-1] requires a momentum resolution $p_{i+1} / p_{i} \lesssim 1.05$, so we would need $\sim 250$ momentum bins (to say nothing of the spatial resolution issues emphasized earlier). Thus, the cost of following the electrons would exceed the cost of the MHD by at least an order of magnitude. Since these are computationally expensive simulations anyway, especially in $3 \mathrm{D}$, that would make ordinary schemes completely impractical at present.

\section{REFERENCES}

Axford, W. I., 1984, in "Magnetic Reconnection in Space and Laboratory Plasmas", ed: E.,W. Hones, (Washington, D.C., American Geophysical Union), p 1

Berezhko, E., Ksenofontov, L. \& Yelshin 1995, Nuclear Physics B, 39A, 171

Biretta, J. A., Stern, C. P. \& Harris, D. E. 1991, AJ, 101, 1632

Blandford, R. D., \& Eichler, D., 1987, Phys. Rept., 154, 1

Borovsky, J. O. \& Eilek, J. A. 1986, ApJ, 308, 929

Bridle, A. H. 1992, in "Testing the AGN paradigm: College Park, MD, 1991", ed: S. Holt, S. G. Neff \& C. M. Urry, (New York: American Institute of Physics), p. 386

Clarke, D. A., Burns, J. O. \& Feigelson, E. D. 1986, ApJ, 300, L41 
Clarke, D., Burns, J. \& Norman, M. 1989, ApJ, 342, 700

Clarke, D. A. 1996, in "Energy Transport in Radio Galaxies and Quasars", A.S.P. Conference Series, Vol 100, ed: P. E. Hardee, A. H. Bridle \& J. A. Zensus, (San Francisco: Astronomical Society of the Pacific), p 311

Coleman, C. S. \& Bicknell, G. V. 1988, MNRAS, 230, 497

Courant, R., \& Friedrichs, K. O. 1948, "Supersonic Flow and Shock Waves", (New York: Springer-Verlag)

Dennett-Thorpe, J., Bridle, A. H., Scheuer, P. A. G., Laing, R. A. \& Leahy, J. P. 1997, MNRAS, 289,753

Drury, L. O'C. 1983, Rept. Prog. Phys., 46, 973

Drury, L. O'C. 1991, MNRAS, 251, 340

Eilek, J. A., Melrose, D. B. \& Walker, M. A. 1997, ApJ, 483, 282

Ellison, D. C., Giacalone, J., Burgess, D. \& Schwartz, S. J. 1993, JGR, 98, 21085

Evans, C. R., \& Hawley, J. F. 1988, ApJ, 332, 659

Falle, S. A. E. G. \& Giddings, J. 1987, MNRAS, 225, 399

Grappin, R., Frisch, U., Pouquet, A. \& Leorat, J. 1982, A\&A, 105, 6

Harten, A. 1983, J. Comp. Phys. 49357

Heavens, A. F. \& Meisenheimer, K., 1987, MNRAS, 225, 335

Jaffe, W. J. \& Perola, G. C. 1973, Ast. \& Ap., 26, 423

Jokipii, J. R. 1987, ApJ, 313, 842

Jones, T. W., Gaalaas, J. B., Ryu, D. \& Frank, A. 1997, ApJ, 482, 230

Jones, T.W. \& Kang, H. 1993, ApJ, 402, 560

Jones, T. W., O’Dell, S. L. \& Stein, W. A. 1974, ApJ, 188, 353

Jones, T. W., Kang, H. \& Tregillis, I. L. 1994, ApJ, 432, 194

Jones, T. W., Ryu, D. \& Frank, A. 1998, in "Numerical Astrophysics 1998" , Tokyo Meeting Proceedings (in press)

Jun, B.-I. \& Jones, T. W. 1998, ApJ(submitted)

Kang, H. \& Jones, T. W. 1991, MNRAS, 249, 439 
Kang, H. \& Jones, T. W. 1995, ApJ, 447, 944

Kang, H. \& Jones, T. W. 1997, ApJ, 476, 875

Kang, H., Jones, T. W. \& Ryu, D. 1992, ApJ, 385, 193

Kardashev, N. S. 1962, Sov. Ast. - AJ, 6, 3

Katz-Stone, D., Rudnick, L. \& Anderson, M. 1993, ApJ, 407, 549

Krülls, W. M. 1992, Ast. \& Ap, 260, 49

Lagage, P. O. \& Cesarsky, C. J. 1983, A\&A, 125, 249

Leahy, J. P., Black, A. R. S., Dennett-Thorpe, J. Hardcastle, M. J. Komissarov, S., Perley, R. A., Riley, J. M. \& Scheuer, P. A. G. 1997, MNRAS, 291, 20

Lee, M. A. \& Völk H. J. 1973, Ast. \& Space Sci., 24, 31.

Levinson, A. 1996, MNRAS, 278, 1018

Lind, K., Payne, D., Meier, D., \& Blandford, R. 1989, ApJ, 344, 89

Mack, K.-H., Klein, U., O’Dea, C. P., Willis, A. G. \& Saripalli, L. 1998, Astr. \& Ap., 329, 431

Matthews, A. P. \& Scheuer, P. A. G. 1990, MNRAS, 242, 616

Meisenheimer, K., Röser, H.-J. \& Schlötelburg, M. 1996, Astr. \& Ap., 307, 61

Miniati, F., Jones, T. W. \& Ryu, D. 1998, ApJ, (submitted)

Myers, S. T. \& Spangler, S. R. 1985, ApJ, 291, 52

Norman, M.L. 1993, in "Energy Transport in Radio Galaxies and Quasars", A.S.P. Conference Series, Vol 100, ed: P. E. Hardee, A. H. Bridle \& J. A. Zensus, (San Francisco: Astronomical Society of the Pacific), p 319

Norman, M. L., Smarr, L. \& Winkler, k.-H. A. 1985, in "Numerical Astrophysics", ed. J. Centrella, J. LeBlanc, \& R. Bowers (Boston: Jones \& Bartlett), p. 88

Rudnick, L. \& Katz-Stone, D. M. 1996, in "Energy Transport in Radio Galaxies and Quasars", A.S.P. Conference Series, Vol 100, ed: P. E. Hardee, A. H. Bridle \& J. A. Zensus, (San Francisco: Astronomical Society of the Pacific), p 233.

Ryu, D. \& Jones, T. W. 1995, ApJ, 442, 228

Ryu, D., Jones, T. W. \& Frank, A. 1995, ApJ, 452, 785

Ryu, D., Miniati, F., Jones, T. W. \& Frank, A. 1998, ApJ(in press) 
Ryu, D., Yun, H., \& Choe, S. 1995, Journal of the Korean Astronomical Society, 28, 223

Ryu, D., Miniati, F., Jones, T. W. \& Frank A. 1998, ApJ, (in press).

Ryu, D. Jones, T. W. \& Frank, A. 1998, (in preparation)

Sato, T. \& the Complexity Simulation Group 1996, Phys. Plasmas (3), 5, 2135

Skilling, J. 1975, MNRAS, 172, 557

Sparks, W. B., Biretta, J. A. \& Macchetto, F. 1996, ApJ, 473, 254

Takarada, K. 1989, P.A.S.J., 41, 927

Treichel, K. \& Rudnick, L. 1998 (in preparation)

Weiss, N. O. 1966, Proc. R. Soc. Lond., A, 293, 310

This preprint was prepared with the AAS LATEX macros v4.0. 


\section{FIGURE CAPTIONS}

Fig. 1 Dynamical flow properties of the simulated MHD jet at $t=10.67$. Normal gray scale is used, with high tones representing high values. Shown top to bottom are: (a) Log gas density, (b) Log magnetic pressure, (c) Gas compression, with shocks showing high tones.

Fig. 2 (a,b): Log synchrotron emissivity computed at $1.4 \mathrm{GHz}$ from the simulated electron distributions for Models $\mathbf{1}$ and $\mathbf{2}$ at the same time and with the same MHD properties as in Fig. 1. (c): "Pseudo emissivity", $j_{c}$, using only MHD variables. The same dynamic range is shown for all; namely, $3 \times 10^{3}$ below the maximum value in each case.

Fig. 3 Synchrotron emissivity spectral index distributions, $\alpha_{1}$ and $\alpha_{2}$, for the electron acceleration Models 1 and 2, as shown in Fig. 2. (a) Model 1, (b) Model 2. Regions are included only if they show in Fig. 2 and have $\alpha \leq 0.8$.

Fig. 4 (a): Log synchrotron emissivity at $1.4 \mathrm{GHz}$ computed from Model 3, which includes strong synchrotron aging effects. The dynamic range is increased over Fig. 2 by a factor 10 to $3 \times 10^{4}$ to capture the same physical regions. (b): Synchrotron emissivity spectral index distribution, $\alpha_{3}$ for Model 3. Regions are included only if they show in Fig. 4a. (c): Synchrotron spectral curvature between $1.4 \mathrm{GHz}$ and $5 \mathrm{GHz}$ for the same regions shown in Fig. 4b.

Fig. 5 Scatter plot displaying the distribution of spectral index vs emissivity [(a), (c), (e)] and spectral magnetic field vs index [(b), (d), (f)] values for Models 1, 2 and $\mathbf{3}$ at $t=10.67$ Each point represents one zone on the simulation grid, using a stride of 2 to reduce the size of the plot file. The number of points in a given range on these plots can be seen as the area on the computational grid with those properties. The magnetic field is normalized by $B_{\text {background }}$.

Fig. 6 A 2-D test calculation of the relativistic electron transport scheme described in section A.3. It involves the evolution of two obliquely intersecting shocks, as described fully in the text. The computational box was given dimensions $1.5 \times 1.5$ and the time shown is $t=0.05$.

(a) Gas density, with the five dynamical flow regions labeled. High tones are high densities.

(b) Electron momentum spectral index. High tones are flatter spectra.

Fig. 7 Tests of the electron transport scheme based on simulations identical to Model 3 (see Table 1), except these were done at half the spatial resolution. Gray scale image: Log of the relativistic electron density. (a),..,(d): Computed electron momentum distributions at indicated locations using 4 (triangle), 8 (star), and 12 (square) momentum bins to cover the range shown. The plot (b) was taken from a region subject only to synchrotron aging effects, and shows the results when $t=\tau_{\text {so }}$ (see eq. [3-1], with $\hat{p}=10^{4}$, along with the analytic solution (solid curve). 
This figure "fig1a.gif" is available in "gif" format from: http://arxiv.org/ps/astro-ph/9809081v1 
This figure "fig2a.gif" is available in "gif" format from: http://arxiv.org/ps/astro-ph/9809081v1 
This figure "fig3a.gif" is available in "gif" format from: http://arxiv.org/ps/astro-ph/9809081v1 
This figure "fig4a.gif" is available in "gif" format from: http://arxiv.org/ps/astro-ph/9809081v1 
This figure "fig5a.gif" is available in "gif" format from: http://arxiv.org/ps/astro-ph/9809081v1 
This figure "fig6a.gif" is available in "gif" format from: http://arxiv.org/ps/astro-ph/9809081v1 
This figure "fig7a.gif" is available in "gif" format from: http://arxiv.org/ps/astro-ph/9809081v1 